\title{
Superradiant Self-Similar Pulses in the Evolution of the Electron-Beam-Plasma Instability
}

\author{
R. Bonifacio, ${ }^{(a)}$ C. Maroli, and N. Piovella \\ Dipartimento di Fisica dell'Università di Milano, Via Celoria 16, 20133 Milano, Italy
}

(Received 12 May 1992)

\begin{abstract}
We derive a set of nonlinear partial differential equations describing the space-time evolution of Langmuir waves in a beam-plasma system, taking into account the propagation effects due to the difference between the beam and wave velocities. We demonstrate the existence of superradiant spikes behind the electron beam front that can be represented by a self-similar solution of the system of equations. These spikes are associated with self-bunching in the electron beam. The conditions in which the predicted spiking behavior could be experimentally observed are discussed.
\end{abstract}

PACS numbers: $52.35 . \mathrm{Qz}$

The beam-plasma instability is certainly one of the most familiar instabilities in plasma physics. Substantial progresses in the understanding of the saturation process and basic nonlinear effects in the evolution of this instability were initiated with the single-wave hypothesis proposed by Drummond et al. in 1970 [1]. According to this hypothesis, the most unstable wave grows exponentially from the background plasma noise, until a large amplitude signal, characterized by a very narrow frequency bandwidth, is created so that the electrons of the beam interact with a very nearly pure sinusoidal wave. The model treated the background plasma as a linear dielectric and predicted that the saturation in the growth of the wave amplitude was due to the trapping of the electrons of the beam in the potential wells of the wave. In subsequent works, O'Neil, Winfrey, and Malmberg $[2,3]$ were able to write a set of nonlinear equations describing the interaction between a plasma and a small cold beam in a stationary situation. It is of interest to note that the single-wave model equations, as they appear in Ref. [2], are formally identical with those describing a free-electron laser in the Compton regime and in a steady-state situation [4], i.e., when one can neglect all "slippage effects" due to the fact that the beam and the radiation are propagating with different velocities. The generalization of the equations written in Ref. [2] to the case of relativistic electron beams was taken up by Matsiborko et al. [5] and Lampe and Sprangle [6]. When the beam strength exceeds a definite threshold [7], the strong Langmuir wave is able to interact nonlinearly also with the electrons of the background plasma, producing cavities in the electron density and giving rise to localized spikes in the electric field of the wave [8].

In this paper we wish to extend the equations written for a small cold beam in Refs. [2] and [6] to the case in which the slippage between the electron beam and the Langmuir signal is taken into account. We are able to demonstrate the existence of a new kind of superradiant self-similar spike of the electric field amplitude developing in that region of the electron pulse that we shall call the "slippage region." The emission mechanism prevailing in this region, which is immediately behind the electron pulse front as it propagates through the plasma, is different from that prevailing in the main body of the pulse (steady-state region) as it has been described in Refs. [2, 3]. In the slippage region, the Langmuir field has a dependence on both space and time and it develops into a superradiant spike [9] that continues to grow and narrow as it propagates along the beam, until the plasma dynamics comes into play and the Langmuir wave collapses [7]. The superradiant spike is not trapping the electrons of the beam and is, therefore, able to extract energy from them with the maximum of efficiency.

We treat the plasma ions as a stationary neutralizing background and the electrons of the plasma as a nearly cold linear dielectric and restrict our analysis to a onedimensional $(z, t)$ situation. If the current densities of the plasma and of the electron beam are $J_{p}(z, t)$ and $J_{b}(z, t)$, respectively, the electric field $E(z, t)$ of the wave excited in the beam-plasma system obeys the equation

$$
\frac{\partial E(z, t)}{\partial t}=-4 \pi\left[J_{p}(z, t)+J_{b}(z, t)\right]
$$

whereas the plasma current density $J_{p}(z, t)$ is governed by the equation

$$
\frac{\partial^{2} J_{p}}{\partial t^{2}}-3 v_{T}^{2} \frac{\partial^{2} J_{p}}{\partial z^{2}}=\frac{\omega_{p}^{2}}{4 \pi} \frac{\partial E}{\partial t}
$$

where $v_{T}=\sqrt{T / m}$ is the plasma thermal velocity, $\omega_{p}=\sqrt{4 \pi e^{2} n_{p 0} / m}$ is the plasma frequency, and $n_{p 0}$ is the unperturbed plasma electron density.

It is convenient to write the field and the current densities as

$$
\begin{aligned}
& E(z, t)=E_{0}(z, t) e^{i(k z-\omega t)}+\text { c.c. } \\
& J_{p}(z, t)=J_{p 0}(z, t) e^{i(k z-\omega t)}+\text { c.c. }, \\
& J_{b}(z, t)=J_{b 0}(z, t) e^{i(k z-\omega t)}+\text { c.c. }
\end{aligned}
$$

where $\omega=\sqrt{\omega_{p}^{2}+3 v_{T}^{2} k^{2}}$. We assume the complex amplitudes $E_{0}, J_{p 0}$, and $J_{b 0}$ to vary slowly in space and time, i.e., $\left|\partial E_{0} / \partial t\right| \ll \omega\left|E_{0}\right|$ and $\left|\partial E_{0} / \partial z\right| \ll k\left|E_{0}\right|$, and similar expressions for the current density amplitudes [slowly 
varying envelope approximation (SVEA)]. This hypothesis is consistent with the assumptions, in the single-wave model of Refs. [2,3], of small, fast, and nearly cold electron beam, $v_{T} / v_{b} \ll\left(n_{b 0} / n_{p 0}\right)^{1 / 3} \ll \gamma_{b}$, where $n_{b 0}$ is the beam density and $\gamma_{b}=\left(1-v_{b}^{2} / c^{2}\right)^{-1 / 2}$ is the energy in $m c^{2}$ units of the electrons of the beam with velocity $v_{b}$. The excited large amplitude signal has a very narrow bandwidth around the wave number $\omega / v_{b}$.

By substituting (3) into Eqs. (1) and (2) we obtain

$$
\begin{aligned}
& \frac{\partial E_{0}}{\partial t}-i \omega E_{0}=-4 \pi\left(J_{p 0}+J_{b 0}\right), \\
& \left(\frac{\partial^{2}}{\partial t^{2}}-3 v_{T}^{2} \frac{\partial^{2}}{\partial z^{2}}\right) J_{p 0}-2 i \omega\left(\frac{\partial}{\partial t}+v_{g} \frac{\partial}{\partial z}\right) J_{p 0} \\
& =-\omega_{p}^{2} J_{b 0},
\end{aligned}
$$

where $v_{g}=3 v_{T}^{2} k / \omega$ is the group velocity of the Langmuir wave in the plasma. Making use of the SVEA approximation and assuming $k v_{T} / \omega_{p} \ll 1$, we obtain

$$
\begin{aligned}
\left(\frac{\partial}{\partial t}+v_{g} \frac{\partial}{\partial z}\right) E_{0} & =-2 \pi\left(\omega_{p} / \omega\right)^{2} J_{b 0} \\
& =-2 \pi\left(\omega_{p} / \omega\right)^{2}\left\langle J_{b}(z, t) e^{-i(k z-\omega t)}\right\rangle,
\end{aligned}
$$

where on the right-hand side of Eq. (6) we used Eq. (3c) and averaged over a wavelength $\lambda=2 \pi / k$. Since $E_{0}$ is slowly varying on the scale of a wavelength, it can only be driven by a beam current averaged over a distance $l$ several wavelengths long. We now assume that the beam consists of $N$ charge sheets with positions $z_{j}(t)$, where $j=1,2, \ldots, N$, and write Eq. (6) in the following form:

$\left(\frac{\partial}{\partial t}+v_{g} \frac{\partial}{\partial z}\right) E_{0}=2 \pi e\left(\omega_{p} / \omega\right)^{2} \frac{n_{b 0}}{N} \sum_{j=1}^{N} v_{j} e^{-i \theta_{j}}$,

where $v_{j}=d z_{j} / d t$ is the velocity of the $j$ th sheet and $\theta_{j}=k z_{j}(t)-\omega t=k\left[z_{j}(t)-v_{r} t\right]$, where $v_{r} \equiv \omega / k$ is the resonant velocity. The dynamics of the electrons in the $j$ th sheet can be approximately described by the equations

$$
\begin{aligned}
& \left(\frac{\partial}{\partial t}+v_{b} \frac{\partial}{\partial z}\right) \theta_{j}=k\left(v_{j}-v_{r}\right) \\
& \left(\frac{\partial}{\partial t}+v_{b} \frac{\partial}{\partial z}\right) v_{j}=-\frac{e}{m \gamma_{j}^{3}}\left[E_{0} e^{i \theta_{j}}+\text { c.c. }\right] .
\end{aligned}
$$

In deriving these last two equations we supposed the electron distribution to vary slowly in space on the scale of a wavelength, and the envelope of the electron current to travel at the constant velocity $v_{b}$.

The preceding basic equations (7), (8), and (9) can be conveniently written in the following final form:

$$
\begin{aligned}
& \frac{\partial \theta_{j}}{\partial \bar{z}}=p_{j} \\
& \frac{\partial p_{j}}{\partial \bar{z}}=-\left[1-2 S p_{j}\left(1+\rho p_{j} / 2\right)\right]^{3 / 2}\left(A e^{i \theta_{j}}+\text { c.c. }\right),
\end{aligned}
$$

$$
\left(\frac{\partial}{\partial \bar{z}}+\frac{\partial}{\partial \tau}\right) A=\chi(\tau) \frac{1}{N} \sum_{j=1}^{N}\left(1+\rho p_{j}\right) e^{-i \theta_{j}},
$$

where $p_{j}=\left(v_{j}-v_{r}\right) / \rho v_{r}, A=E_{0} / \sqrt{2 \pi m v_{r}^{2} \bar{n}_{b 0} \gamma_{r}^{3} \rho \beta_{g}}$, $\bar{z}=k \rho\left(v_{r} / v_{b}\right) z$, and $\tau=\omega \rho\left(t-z / v_{b}\right) /\left(\beta_{g}-1\right)$, with $\beta_{g}=v_{b} / v_{g}$. Equations (10)-(12) depend only on two parameters, $\rho=\left(1 / \gamma_{r}\right)\left(\bar{n}_{b 0} \beta_{g} / 2 n_{p 0}\right)^{1 / 3}\left(\omega_{p} / \omega\right)^{4 / 3}$ and $S=\rho \beta_{r}^{2} \gamma_{r}^{2}$, where $\beta_{r}=v_{r} / c$ and $\gamma_{r}=\left(1-\beta_{r}^{2}\right)^{-1 / 2}$. Note that we introduced in Eq. (12) a longitudinal electron beam profile, $n_{b 0}(\tau)=\bar{n}_{b 0} \chi(\tau)$, where $\bar{n}_{b 0}$ is the peak value of the beam density and $\chi(\tau)$ is normalized to 1 .

Equations (10)-(12) extend the previous steady-state model equations of Refs. [2,3] and their relativistic generalization $[5,6]$ to the case in which the slippage between the electron beam and the wave is taken into account. The two preceding models assumed the plasma and the electron beam to be only one wavelength long with periodic boundary conditions at each end. This can be appropriate only for an infinitely long electron pulse and/or a continuous excitation at the border of the plasma. The periodic boundary condition assumptions are not appropriate when one wishes to examine how the front of the electron pulse propagates into the plasma, or when the source of the perturbation, which is amplified by the beam-plasma instability, has a pulsed character. In these situations, no steady-state evolution is possible behind the electron beam front or of the excitation pulse.

In order to simplify the analysis of Eqs. (10)-(12), we consider only the limit case in which $\rho\left|p_{j}\right| \ll 1$ (i.e., $\mid v_{j}-$ $v_{r} \mid \ll v_{r}$ ) and $S\left|p_{j}\right| \ll 1$ (i.e., $\left|\gamma_{j}-\gamma_{r}\right| \ll \gamma_{r}$ ). In these limits the system reduces to

$$
\begin{aligned}
& \frac{\partial \theta_{j}}{\partial \bar{z}}=p_{j}, \\
& \frac{\partial p_{j}}{\partial \bar{z}}=-\left(A e^{i \theta_{j}}+\text { c.c. }\right), \\
& \left(\frac{\partial}{\partial \bar{z}}+\frac{\partial}{\partial \tau}\right) A=\chi(\tau) b(\bar{z}, \tau),
\end{aligned}
$$

where $b(\bar{z}, \tau) \equiv\langle\exp (-i \theta)\rangle=(1 / N) \sum_{j=1}^{N} \exp \left(-i \theta_{j}\right)$ is the bunching parameter, describing the spatial modulation of the beam. Equations (13)-(15) are formally identical to those describing a free-electron laser in the Compton regime [4]. The space and time variables are scaled with respect to the gain length $L_{g}=(\lambda / 2 \pi \rho) v_{b} / v_{r}$ and the cooperation time $T_{c}=\left(\beta_{g}-1\right) L_{g} / v_{b}$, so that $\bar{z}=z / L_{g}$ and $\tau=\left(t-z / v_{b}\right) / T_{c}$.

Before proceeding to investigate the nonlinear evolution of (13)-(15), we summarize the main results $[10,11]$ of a linear analysis around the equilibrium state, with no field and cold, monoenergetic, and unmodulated beam distribution. We will assume henceforth a step profile for the electron beam $\left[\chi(\tau)=1\right.$ for $0<\tau<\tau_{p}$, where $\tau_{p}$ is the duration of the electron pulse, and zero elsewhere] and an external excitation at $\bar{z}=0$, 
$A(0, \tau)=A_{0} \exp (-i \delta \tau)$, of constant amplitude, where $\delta \equiv\langle p\rangle_{0}=\left(v_{b}-v_{r}\right) / \rho v_{r}$ is the detuning parameter. It is possible to demonstrate [10] the existence of two regions in the electron pulse. In the slippage region $(0<\tau<\bar{z})$ behind the beam front and for a time interval equal to the delay due to the slippage between the electrons and the wave, the wave amplitude has both a space and time dependence. In the steady-state region $\left(\bar{z}<\tau<\tau_{p}\right)$ the wave intensity has only a space dependence, growing as $\exp (2|\operatorname{Im} k| \bar{z})$, where $k$ is the root of the cubic equation $k^{2}(k-\delta)+1=0$, which is unstable for $\delta<\delta_{T}=(27 / 4)^{1 / 3}$ with maximum growth rate at $\delta=0$. In the slippage region a growing wave packet appears as the beam moves along the plasma. A detailed study $[11,12]$ shows that in the linear regime the peak of such a wave packet moves at the constant velocity $V_{G}=3 v_{b} /\left(1+2 \beta_{g}\right)$, while its width is increasing in proportion of the square root of $\bar{z}$ and the peak of its intensity is growing exponentially as a function of $\bar{z}$, with the maximum rate. The length of the slippage region, for $v_{b} \gg v_{T}$, is practically equal to the plasma length, so that, for electron pulses shorter than the plasma column, the slippage region covers the whole beam.

As described in Refs. [2,3] and experimentally observed $[13,14]$, the amplitude of the wave in the steady-state region grows exponentially in the linear regime, until, after some distance from the point where the beam enters the plasma, the wave amplitude stops growing and begins to oscillate around a mean value. On the contrary, in the slippage region, the wave packet starts to narrow in the nonlinear regime and evolves into a superradiant, self-similar spike. In both cases the interaction produces self-bunching in the electron beam distribution, with $|b| \sim 0.8$ at the peak. In Fig. 1 we show the wave amplitude as a function of $\tau$ at $\bar{z}=30$, as it results from the numerical integration of Eqs. (13)-(15), with $A_{0}=10^{-4}$, (a) $\delta=0$, and (b) $\delta=4$, and for an electron beam of duration $\tau_{p}=50$. In the steady-state region $\bar{z}<\tau<\tau_{p}$, the wave amplitude is constant, whereas in the slippage region $0<\tau<\bar{z}$ we observe the growing of a large spike. In the region of free propagation behind the trailing edge of the beam, $\tau_{p}<\tau<\tau_{p}+\bar{z}$, and in the resonant case [Fig. 1(a)], we can see the track of the spatial evolution between $\bar{z}=0$ and $\bar{z}=30$. In the detuned case [Fig. 1(b)] no appreciable growth of the wave amplitude occurs in the steady-state region, as the perturbation is stable for $\delta>\delta_{T}$, whereas in the slippage region the wave packet has evolved into a large spike. A numerical study shows that the spike continues to grow and narrow as it propagates along the plasma, slipping behind the beam front. Observing the evolution of the spike along the plasma in a coordinate frame moving at the velocity $v_{b}$ of the beam (i.e., at $\tau$ constant) and comparing the spike at different values of $\tau$ (i.e., for different distances from beam front), we find that the peak amplitude and the width of the spike are,
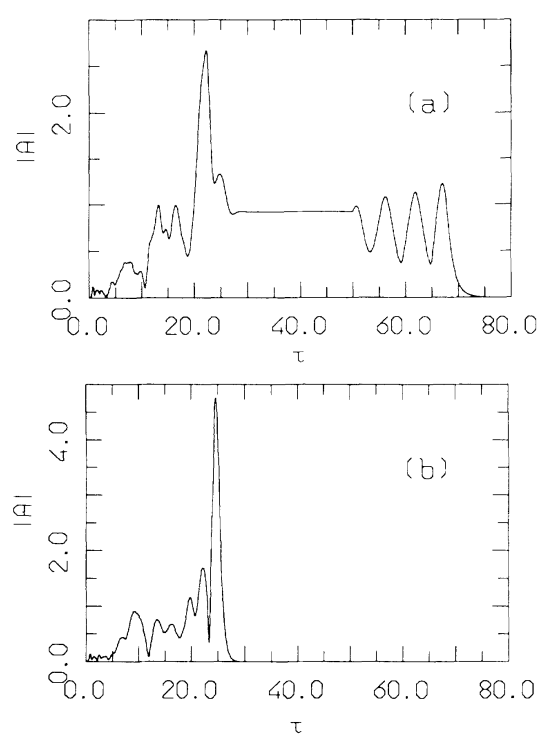

FIG. 1. Normalized wave amplitude $|A|$ vs $\tau$, from numerical integration of Eqs. (13)-(15), for a fixed position along the plasma, $\bar{z}=30, A_{0}=10^{-4}$, and an electron pulse of duration $\tau_{p}=50$; (a) resonant case $\delta=0$; (b) detuned case $\delta=4$.

respectively, proportional to $\tau$ and $\tau^{-1 / 2}$. Such a spike is described [11] by the following exact self-similar solution of the partial differential equations (13)-(15), in the case of constant beam profile $[\chi(\tau)=1]: A(\bar{z}, \tau)=\tau A_{1}(y)$ and $\theta_{j}(\bar{z}, \tau)=\theta_{1 j}(y)$, where $y=\sqrt{\tau}(\bar{z}-\tau)$. Once substituted into Eqs. (13)-(15), this particular solution reduces the partial differential equations to a system of ordinary differential equations for the variables $\theta_{1 j}$ and $A_{1}$ :

$$
\begin{aligned}
& \frac{d^{2} \theta_{1 j}}{d y^{2}}=-\left(A_{1} e^{i \theta_{1 j}}+\text { c.c. }\right), \\
& (y / 2) \frac{d A_{1}}{d y}=\left\langle e^{-i \theta_{1}}\right\rangle-A_{1} .
\end{aligned}
$$

These equations give a good description of the Langmuir wave packet even when the beam profile $\chi(\tau)$ is not constant but slowly varying with respect to the width of the spike, at least in the asymptotic limit in which the selfsimilar solution strictly applies. Equations (16) and (17) do not contain explicitly the variable $\tau$ : It follows, therefore, that the peak amplitude of the wave is proportional to $\tau$ and, from the universal scaling, $\left|E_{0}\right|^{2} \propto n_{b}^{2}$, i.e., that the field in the pulse is superradiant. The width of the pulse is inversely proportional to $\sqrt{\tau}$, i.e., inversely proportional to the square root of the peak amplitude. The nonstationary emission in the slippage region behind the beam front or, more generally, a pulsed perturbation, generates in the linear stage of the evolution a wave packet that evolves in the nonlinear regime toward the superradiant self-similar solution. Whereas a constant perturbation in a continuous beam gives rise to a stationary emission that stops when the electrons be- 


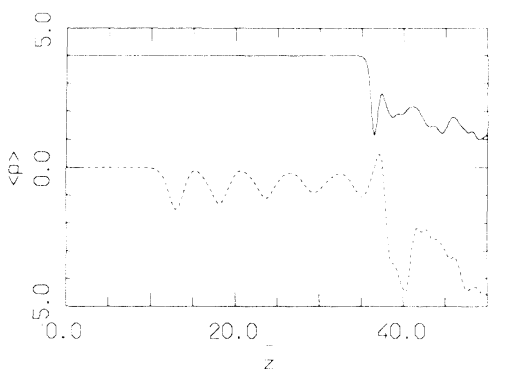

FIG. 2. Normalized average beam velocity $\langle p\rangle$ vs $\bar{z}$, at $\tau=30$, for $A_{0}=10^{-4}, \delta=0$ (dashed line), and $\delta=4$ (continuous line).

come trapped by the large wave, the superradiant spikes generated in the slippage region of the beam continue to grow without trapping the electrons and extracting energy from them. That the trapping process is not relevant for the superradiant emission can be shown in Fig. 2 , where the scaled average beam velocity $\langle p\rangle$ is plotted as a function of the position $\bar{z}$ along the plasma, at $\tau=30$ for the cases of Figs. 1(a) and 1(b). In the resonant case (dashed line), the electrons at the selected position $\tau$ in the beam perform synchrotron oscillations due to the trapping by the steady-state wave, until (for $\tau>\bar{z}$ ) they interact with the large superradiant spike propagating from the leading edge and lose a considerable fraction of their energy. After the interaction with the spike, the electrons are left completely untrapped and with a large amount of energy spread. The detuned case (continuous line) shows that the same process also takes place if the electrons are untrapped before the arrival of the spike. Hence, the slippage inhibits the trapping of the beam electrons, which is responsible for both the saturation mechanism and the development of the sideband instability. The optimum extraction of energy occurs when the electrons undergo half an oscillation in the potential well of the superradiant wave. As the oscillation frequency is proportional to the square root of the wave amplitude, the distance in which the electrons undergo half an oscillation decreases as the wave intensity becomes higher. As a consequence, the width of the spike decreases in proportion to the square root of its peak amplitude. The condition that the electrons undergo half an oscillation in the potential can be maintained at an arbitrarily large peak intensity, provided that the wave pulse is sufficiently short. This is because the electrons do not remain in the pulse, but slip forward through it.

The superradiant spiking behavior is observable when the strength of the Langmuir wave is below the threshold of collapse [7] $\left[\left|E_{0}\right|^{2} / 8 \pi n_{p 0} T \gg\left(k \lambda_{D}\right)^{2}\right.$, or, using the scaled variables, $\beta_{g}\left(\mathcal{E}_{e} / T\right) \rho^{4}|A|^{2} \gg 10$, where $\lambda_{D}$ is the Debye length and $\mathcal{E}_{e}=m v_{b}^{2} / 2$ is the beam energy in $\mathrm{eV}$. The predicted superradiant spikes and their temporal and spatial evolution could be experimentally observed by performing, for instance, an experiment of the same type as described in Refs. $[13,14]$. In this case, the plasma has a density $n_{p 0} \sim 10^{10} \mathrm{~cm}^{-3}$ and a temperature $T \sim 10 \mathrm{eV}$. An electron beam is injected, with $\bar{n}_{b 0} / n_{p 0} \sim 10^{-3}$ and with $v_{b} / v_{T} \sim 5$. With the preceding values, one has $\rho \sim 0.14, L_{g} \sim 0.7 \mathrm{~cm}$, and $T_{c} \sim 8 \mathrm{~ns}$ and $\beta_{g}\left(\mathcal{E}_{e} / T\right) \rho^{4}|A|^{2} \sim 0.04$. In this range of parameters, as seen by Gentle and co-workers $[13,14]$ in stationary conditions, there are no other processes, like parametric decays or weak turbulence phenomena [15], that may compete with the development of superradiant spikes.

In conclusion, we have shown that, in the evolution of the beam-plasma instability, space-time effects due to the different velocity of propagation of the beam and the wave give rise to a superradiant emission of self-similar spikes. This is the only dynamical regime possible when the electron pulses are shorter than the plasma length.

(a) Also at Istituto Nazionale di Fisica Nucleare, Sezione di Milano, via Celoria 16, 20133, Milano, Italy.

[1] W.E. Drummond, J.H. Malmberg, T.M. O'Neil, and J.R. Thompson, Phys. Fluids 13, 2422 (1970).

[2] T.M. O'Neil, J.H. Winfrey, and J.H. Malmberg, Phys. Fluids 14, 1204 (1971).

[3] T.M. O'Neil and J.H. Winfrey, Phys. Fluids 15, 1514 (1972).

[4] R. Bonifacio, C. Pellegrini, and L. Narducci, Opt. Commun. 50, 373 (1984).

[5] N.G. Matsiborko, I.N. Onishchenko, V.D. Shapiro, and V.I. Shevchenko, Plasma Phys. 14, 591 (1972).

[6] M. Lampe and P. Sprangle, Phys. Fluids 18, 475 (1975).

[7] E. Zakharov, Zh. Eksp. Teor. Fiz. 62, 1745 (1972) [Sov. Phys. JETP 35, 908 (1972)].

[8] A.Y. Wong and B.H. Quon, Phys. Rev. Lett. 34, 1499 (1975).

[9] R. Bonifacio, B.W.J. McNeil, and P. Pierini, Phys. Rev. A 40, 4467 (1989).

[10] R. Bonifacio, C. Maroli, and N. Piovella, Opt. Commun. 68, 369 (1988).

[11] R. Bonifacio, L. De Salvo Souza, P. Pierini, and N. Piovella, Nucl. Instrum. Methods Phys. Res., Sect. A 296, 358 (1990).

[12] M.E. Jones, D.S. Lemons, and M.A. Mostrom, Phys. Fluids 26, 2784 (1983).

[13] K.W. Gentle and C.W. Roberson, Phys. Fluids 14, 2780 (1971).

[14] K.W. Gentle and J. Lohr, Phys. Fluids 16, 1464 (1973).

[15] R.N. Sudan, in Handbook of Plasma Physics, edited by M.N. Rosenbluth and R.Z. Sagdeev (North-Holland, Amsterdam, 1984), Vol. 2, Chap. 6.3, p. 337. 